\title{
Core Competency Education in Communication and Systems-based Practice: Are We Failing Our Residents?
}

\author{
${ }^{1}$ Anthony Alessi, ${ }^{2}$ Marina Boruk
}

\begin{abstract}
Core competencies clearly established by the Accreditation Council for Graduate Medical Education (ACGME) for residency training include 'Systems-based practice' and 'Interpersonal and communication skills'. However, the practical skills of medical documentation and coding that fall under these categories are often ignored in the formal training curriculum as well as during trainee assessments. Residents and practicing physicians alike have been shown to be poor medical coders. The few small studies that have been performed suggest that a combination of formalized lectures and individualized feedback on resident charts may be effective at teaching residents how to properly document and code. In today's health care environment with increasing cost and trends for decreasing reimbursement these skills are essential for the survival of any practicing physician and more effort should be made to ensure that residents are competent in medical documentation and coding.
\end{abstract}

Keywords: Billing, Coding, Core competencies, Medical documentation, Residency training curricula.

How to cite this article: Alessi A, Boruk M. Core Competency Education in Communication and Systems-based Practice: Are We Failing Our Residents? Int J Head Neck Surg 2016;7(1):13-16.

Source of support: None

Conflict of interest: None

\section{INTRODUCTION}

In 1998, The Accreditation Council for Graduate Medical Education (ACGME) began an initiative, known as the outcome project, to improve resident training in our ever evolving health care system. ${ }^{1}$ This initiative defined six 'core competencies' which residents are expected to become proficient in by the end of their training. We would like to focus on two of the six. The 'Systems-based practice' competency requires that resident physicians be able to incorporate cost awareness seamlessly with patient care. However, there are no published references

\footnotetext{
${ }^{1}$ Resident, ${ }^{2}$ Assistant Professor

1,2Department of Otolaryngology Head and Neck Surgery, SUNY Downstate Medical Center, Brooklyn, New York, USA
}

Corresponding Author: Anthony Alessi, Resident, Department of Otolaryngology Head and Neck Surgery, SUNY Downstate Medical Center, 450 Clarkson Ave, Box 126, Brooklyn New York USA, Phone: 718-270-1638, Fax: 718-270-3924 e-mail: anthony.alessi@downstate.edu for such costs that widely differ by insurance carrier, region, and varying often strict guidelines, leaving physicians to learn by 'trial and error'. The 'Interpersonal and communication skill' competency expects that resident physicians be able to maintain accurate medical records and communicate effectively with not only other health care providers but also with health-related agencies, such as health care insurers. Despite these requirements by the ACGME, few residency programs in the United States have formal curricula designed to teach residents the practical skills of medical coding, billing and documentation. ${ }^{2,3}$ These skills, in today's world of decreasing reimbursement and increasing cost, are vital to the survival and health of a medical practice in any specialty. While the medical literature on this topic is sparse, the literature within the field of otolaryngology and head and neck surgery is almost non-existent. ${ }^{4,5}$ This review will explore the deficiencies in resident education on medical coding based on documentation. We will review published studies where a formalized curriculum was implemented to teach residents practice management skills and attempt to determine how these vital skills can be taught effectively and efficiently in otolaryngology training programs. We would like to explore and promote incorporation of formal education and assessment of medical documentation based on the American Medical Association (AMA) guidelines, coding and billing compliance into the residency curriculum. The implications for a physician not properly trained in the business aspects of medical practice will then be discussed.

The lack of teaching of medical coding and billing during residency is not a new problem. While medical schools and residency programs have excelled at teaching trainees the medical knowledge needed for quality patient care, the business aspect of medicine has often been ignored completely. In a 1981 survey of 717 third year family practice residents, $87 \%$ of residents stated that they had zero training in the business of medicine in both medical school and residency. ${ }^{6}$ Little has been done to correct this problem since that time. A more recent study by Fakhry et al found that while $92 \%$ of surgical residents believed that knowledge of medical coding and billing were important, $85 \%$ felt that their knowledge of those topics was inadequate. ${ }^{7}$ Residents in the fields 
of emergency medicine and obstetrics and gynecology have responded similarly in surveys large and small..$^{8-10}$ Objective studies of physicians' ability to document and code have confirmed what these surveys have long suggested: training of physicians in medical coding and documentation is inadequate. In a blinded study ${ }^{11}$ of 50 resident surgical operative notes, it was found that $30 \%$ of the dictations were improperly documented when compared to attending operative notes. It was calculated that if resident notes were used to justify reimbursement for these procedures $30 \%$ of charges (about $\$ 18,000$ for the 50 procedures) would have been delayed or denied! One would think that attending physicians would be more proficient than residents at coding. However, this does not seem to be the case. A large study of interventional radiologists found that only $44 \%$ of all encounters were properly coded. ${ }^{12}$ The results within otolaryngology are not much different. Nauraei et al found that within their practice, coding errors were found in $24.1 \%$ of encounters, resulting in the loss of hundreds of dollars per patient encounter. ${ }^{5}$ These findings are not surprising when one learns that even trained physicians can only agree on the same current procedural terminology (CPT) code for an encounter about half the time. ${ }^{13}$

The AMA released specialty specific guidelines on required documentation and coding of evaluation and management (E/M) in 1997. However, 19 years later, coding and documentation experts are busier than ever. Courses, as well as lectures offered during annual American Academy of Otolaryngology-Head and Neck Surgery (AAO-HNS) meetings, for general otolaryngologists and subspecialists are packed equally with both seasoned practitioners frustrated by insurance denials and young physicians who are just trying to figure out how to get paid for what they are well-trained to do. One thing is clear: even medically competent, well-trained physicians, are poorly equipped to properly document, code and bill for the services they provide.

The problem faced by physicians who are poorly trained in medical documentation and coding is two-fold. This stems from the two most common errors made during coding: undercoding and overcoding. Undercoding, the more common of the two, results in the loss of revenue for the physician and his/her practice. ${ }^{3,5}$ Holt et al showed that $80 \%$ of all patient encounters were determined to be undercoded after chart review in a primary care practice. $^{3}$ This loss of revenue can be significant. ${ }^{5,11,14}$ Given the trend for decrease in reimbursement for physicians across the board, as well as threat of decrease in funding for resident education, ${ }^{15}$ it may be difficult for physicians to financially sustain their independent practice or justify their contracted salaries if employed without knowledge and adequate education on proper documentation and coding. Overcoding, although less common than undercoding, ${ }^{3,14}$ can result in consequences that are much more severe. Under the patient protection and Affordable Care Act, overpayments (a result of overcoding) made to physicians which are not reported within 60 days of receipt are punishable with hefty fines. ${ }^{16}$ One residency program was forced to implement a medical billing curriculum after facing extensive financial losses due to overcoding errors. ${ }^{14}$ Practitioners may be excluded by certain insurers, including medicaid and medicare. Furthermore, criminal charges can be brought against the physician if fraud is suspected, resulting in fines up to $\$ 25,000$ and/or up to 5 years imprisonment. ${ }^{17}$ Even worse, anyone can report a physician for receiving overpayments and these 'whistleblowers' can receive a share of fines if collected. ${ }^{16}$ While these laws were implemented to penalize practitioners who are knowingly and wrongfully overcharging, physicians who overcharge because of poor coding skills are liable as well. The department of otolaryngology at the University of Mississippi expanded their objective structured clinical exam (OSCE) to include medical coding and identified two cases of 'insurance fraud' by residents on this examination. ${ }^{4}$ This unexpected finding led to incorporation of additional lectures in socioeconomics and ethics in the resident educational program as well as further education on acceptable practice for encounter documentation and billing. The physicians who are trained in documentation and coding and habitually keep meticulous records can better prevent accidental overcharges and protect his/ her practice from false claims.

Multiple residency training programs have attempted to formalize and study the effectiveness of a curriculum to teach residents documentation and coding. Most of these studies are small, lack true control groups and vary in the depth and breadth of the content taught. However, almost all studies show a trend that any type of formalized curriculum appears to improve resident ability in these crucial areas. The simplest intervention reported was done in the department of family practice at the University of Iowa. ${ }^{18}$ In this 6 years study residents participated in an interactive conference on CPT and E/M coding. Random audits of resident charts were performed before and after this conference. Their analysis found that coding skill improved over the course of residents' training regardless of their participation in the conference. While the Iowa study showed minimal benefit from a simple 1 hour conference, interventions with more extensive curricula show more promise. One surgical residency program employed a three pronged approach to teach residents practice management skills. ${ }^{2}$ 
Residents first participated in a series of lectures on various topics including practice management and managed care. Then each resident took part in a series of 1-hour meetings on documentation and coding in which real patient charts were reviewed. Finally, residents took part in a clinic in which after each patient encounter the chart was presented to a coding and compliance manager who evaluated the chart with the resident. It was found by the coding experts that the most common error made during documentation was failure to document adequate review of systems. ${ }^{2,14}$ With this extensive intervention, resident coding accuracy increased from 36 to $88 \%$. Even the Program Director who also participated in this program had a $40 \%$ increase in coding compliance! Fortunately, less extensive programs also show promise. An obstetrics and gynecology program implemented four simple sessions for each resident to attend. ${ }^{10}$ These sessions included overviews of CPT and International Classification of Diseases (ICD) coding and guidelines on documentation. In addition, individual resident documentation and audited resident dictations were reviewed. Sessions were specifically directed at addressing the most common errors seen in resident documentation. After intervention, residents showed significant decrease in overcoding and undercoding and improved billing of procedures. On average, the amount billed per patient encounter rose $10 \%$. A similar curriculum employed by the Pediatric Neurology Department at Boston Children's hospital, ${ }^{14}$ which included structured feedback and audit of resident notes, showed even more promising results as reimbursement increased $38 \%$ per patient encounter or about $\$ 35,000$ per resident/year. Trainees also employed a review of systems template which helped improve documentation accuracy. In addition, it was found that properly documented notes were actually shorter than improperly documented notes. This supports the findings in other studies that templateguided documentation takes no longer than free-hand documentation and may increase accuracy and improve reimbursement generated for each patient encounter. ${ }^{19,20}$ Emergency medicine residents have also been shown to increase productivity after implementation of formal lectures on coding, case-specific feedback on patient charts and distribution of pocket reference cards on medical coding. ${ }^{21}$ These residents increased the percentage of level 5 encounters coded, the amount of relative value units (RVUs) generated per encounter and even the number of patients seen per hour. It should be cautioned, however, that while many studies have shown increased productivity from residents after training in medical coding, one study did show a trend for residents to overcode more often after training. ${ }^{22}$ This study offered financial incentive to the best coding resident, which may have led to this finding, but given the severe repercussions that can be faced for overcoding, steps should be taken to avoid such unintentional outcomes.

Medical documentation and coding is a skill which is poorly taught and often ignored during residency training. Given the growing importance of medical coding for both financial and compliance reasons, ${ }^{16}$ all medical residencies, including those within otolaryngology, should incorporate a formal curriculum to teach residents these vital skills. Ideally, a foundation with overview of third payer system and basic concepts of practice management should be introduced early in medical school followed by general documentation guidelines for medical coding and billing prior to clinical rotations. Although a simple 'SOAP' note may still be often applicable, knowledge of such instances is imperative. Further nuances as well as field specific aspects of documentation and coding can be addressed during the postgraduate years. ${ }^{14}$ More research needs to be done in order to determine the most effective way to teach residents these skills. However, in the few small studies that have been done, formalized lectures with frequent auditing and feedback on residents charts in a clinic setting have been shown to be most effective at teaching residents how to code. ${ }^{2,10,14,21}$ In addition, residents taught in this manner report the highest confidence levels when it comes to coding an encounter. ${ }^{9}$ Frequent formal assessments of residents' competency in coding, possibly through annual OSCEs, ${ }^{4}$ may also be effective at determining areas of weakness in training so that teaching may be directed toward those areas. Regardless of the exact method used, more attention needs to be paid to this topic: the modern physician cannot survive without it.

From a global perspective, early understanding of guidelines, rules and regulations as well as the healthcare structure within which we practice will ensure better compliance. This in-turn would likely decrease the need for costly and disruptive third party audits, and, at least in part, would decrease the ever-soaring cost of healthcare. More importantly, properly educated residents would be prepared for all situations upon graduation, from starting a financially viable and properly reimbursed practice independently to justifying and demonstrating productivity in the employed setting.

\section{REFERENCES}

1. Swing, Susan R. The ACGME outcome project: retrospective and prospective. Med Teacher 2007;29(7):648-654.

2. Jones K, Lebron RA, Mangram A. Practice management education during surgical residency. Am J Surg 2008;196: 878-882. 
3. Holt J, Warsy A, Wright P. Medical decision making: guide to improved CPT coding. South Med J 2010 Apr 1;103(4): 316-312.

4. Franzese CB. Pilot study of an objective structured clinical examination (the Six Pack) for evaluating clinical competencies. Otolaryngol Head Neck Surg 2008 Feb 1;138(2): 143-148.

5. Nouraei SA, O'Hanlon S, Butler CR, et al. A multidisciplinary audit of clinical coding accuracy in otolaryngology: financial, managerial and clinical governance considerations under payment by results. Clin Otolaryngol 2009;34:43-51.

6. Breitwieser D, Adye W, Arvidson M. Resident evaluation of current practice management training. J Fam Pract 1981;13: 1063-1064.

7. Fakhry SM, Robinson L, Hendershot K, et al. Surgical residents' knowledge of documentation and coding for professional services: an opportunity for a focused educational offering. Am J Surg 2007;194:263-267.

8. Howell J, Chisholm C, Clark A, Spillane L. Emergency medicine resident documentation: results of the 1999. American Board of Emergency Medicine in training examination survey. Acad Emerg Med 2000;7:1135-1138.

9. Pines JM, Braithwaite S. Documentation and coding education in emergency medicine residency programs: a national survey of residents and program directors. California J Emerg Med 2004 Jan;5(1):38.

10. AsSanie S, Zolnoun D, Wechter ME, Lamvu G, Tu F, Steege J. Teaching residents coding and documentation: effectiveness of a problem oriented approach. Am J Obst Gynecol 2005 Nov 30;193(5):1790-1793.

11. Novitsky YW, Sing RF, Kercher KW, Griffo ML, Matthews BD, Heniford BT. Prospective, blinded evaluation of accuracy of operative reports dictated by surgical residents. The American Surgeon 2005 Aug 1;71(8):627-632.
12. Duszak R, Blackham WC, Kusiak GM, et al. CPT coding by interventional radiologists: a multi institutional evaluation of accuracy and its economic implications. J Am Coll Radiol 2004;1:734-740.

13. King MS, Lipsky MS, Sharp L. Expert agreement in current procedural terminology evaluation and management coding. Arch Intern Med 2002;162:316-320.

14. Waugh JL. Education in medical billing benefits both neurology trainees and academic departments. Neurol 2014 Nov 11;83(20):1856-1861.

15. IglehartJK. The uncertain future of medicare and graduate medical education. New England J Med 2011 Oct 6;365(14):1340-1345.

16. Chananie SJ, Colangelo N. New requirement for self disclosing CMS overpayments. J Am Col Radiol 2010 Nov 30;7(11):898-899.

17. Protection P, Act AC. Patient protection and affordable care act. Public Law 2010:111-148.

18. Skelly KS, Bergus GR. Does structured audit and feedback improve the accuracy of residents CPT E and M coding of clinic visits? Family Med 2010 Oct 1;42(9):648.

19. Mulvehill S, Schneider G, Cullen CM, Roaten S, Foster B, Porter A. Template-guided versus undirected written medical documentation: a prospective, randomized trial in a family medicine residency clinic. J Am Board of Family Practice 2005 Nov 1;18(6):464-469.

20. Sprtel SJ, Zlabek JA. Does the use of standardized history and physical forms improve billable income and resident physician awareness of billing codes? South Med J 2005;98:524-527.

21. Carter KA, Dawson BC, Brewer K, Lawson L. RVU ready? Preparing emergency medicine resident physicians in documentation for an incentive based work environment. Acad Emerg Med 2009;16:423-428.

22. Gala RB, Chiang S. The impact of a documentation and coding curriculum in an obstetrics and gynecology continuity clinic. Ochsner J 2012 Dec;12(4):354-358. 